\title{
Biasing effects of receptor-ligand complexes on protein-unfolding statistics
}

\author{
Constantin Schoeler, ${ }^{1}$ Tobias Verdorfer, ${ }^{1}$ Hermann E. Gaub, ${ }^{1}$ and Michael A. Nash ${ }^{1,2,3, *}$ \\ ${ }^{1}$ Lehrstuhl für Angewandte Physik and Center for Nanoscience, Ludwig-Maximilians-Universität München, Amalienstr. 54, \\ 80799 Munich, Germany \\ ${ }^{2}$ Department of Chemistry, University of Basel, Klingelbergstrasse 80, 4056 Basel, Switzerland \\ ${ }^{3}$ Department of Biosystems Science and Engineering, Eidgenössische Technische Hochschule Zürich (ETH-Zürich), \\ 4058 Basel, Switzerland
}

(Received 30 May 2016; published 13 October 2016)

\begin{abstract}
Protein receptor-ligand pairs are increasingly used as specific molecular handles in single-molecule proteinunfolding experiments. Further, known marker domains, also referred to as fingerprints, provide unique unfolding signatures to identify specific single-molecule interactions, when receptor-ligand pairs themselves are investigated. We show here that in cases where there is an overlap between the probability distribution associated with fingerprint domain unfolding and that associated with receptor-ligand dissociation, the experimentally measured force distributions are mutually biased. This biasing effect masks the true parameters of the underlying free energy landscape. To address this, we present a model-free theoretical framework that corrects for the biasing effect caused by such overlapping distributions.
\end{abstract}

DOI: 10.1103/PhysRevE.94.042412

\section{INTRODUCTION}

Mechanical forces play an important role in many biological systems. Refolding of individual titin domains is believed to assist in muscle contraction [1], stretching forces expose cryptic binding sites involved in focal adhesions [2], and mechanically stable receptor-ligand pairs govern the assembly of large extracellular machineries and adhesion of bacterial cells to their cellulosic carbon sources $[3,4]$. Single-molecule pulling experiments with atomic force microscopes [5], optical tweezers [6], or magnetic tweezers [7] have become widely used techniques to study such phenomena at the singlemolecule level.

Due to the stochastic nature of domain unfolding, typical atomic force microscopy experiments record many thousands of data traces to obtain statistically meaningful results from single-molecule pulling experiments. To unambiguously identify the unfolding signals of a given protein domain of interest or the dissociation of a receptor-ligand system under external load, the resulting data sets need to be filtered, and specific, single-molecule interactions must be identified.

To accommodate this need, the community has developed an elegant strategy to achieve high yields of specific curves: In this approach, protein domains of interest are fused to a receptor complex that serves as a specific handle in pulling experiments. This improves curve yields and data fidelity by providing a specific molecular interaction handle to "grab" the protein of interest. Thereby, the unfolding of individual domains and the dissociation of a receptor-ligand complex can be studied in a single experiment [3,4,8-10]. Also, using a known protein domain in the fusion construct provides a unique unfolding pattern that can be used to identify specific traces, when receptor-ligand unbinding itself is studied. These domains are then referred to as fingerprint domains [5].

In order for a curve to be unambiguously identified as constituting specific signal, it needs to exhibit unfolding of all

\footnotetext{
*michael.nash@unibas.ch
}

included fingerprint domains plus a specific receptor-ligand dissociation event. Throughout this letter we will refer to domains fused to a receptor-ligand complex as fingerprint domains for both scenarios, namely protein-unfolding studies using receptor-ligand complexes as specific handles, as well as unbinding studies of receptor-ligand complexes of interest, which use fingerprint domains for assistance in data filtering. We discuss the statistical effects that arise when the respective force distributions for fingerprint domain unfolding and receptor-ligand complex rupture exhibit a finite overlap. We quantitatively show how the statistical overlap between these two distributions affects the experimentally observable unfolding and rupture force distributions. We provide a framework for extracting kinetic and energetic information from the experimentally observed distributions that are corrected for the biasing effects arising from such overlaps in a model-free fashion.

\section{THEORETICAL FRAMEWORK}

The standard theoretical framework treats protein unfolding or bond dissociation as thermally driven escape over a free energy barrier that is modulated by an external force $F$ [11-14]. This description leads to a general expression for the distribution of unfolding or rupture forces in a pulling experiment,

$$
p(F)=\frac{k(F)}{\dot{F}(F)} \exp \left(-\int_{0}^{F} \mathrm{~d} F^{\prime} \frac{k\left(F^{\prime}\right)}{\dot{F}\left(F^{\prime}\right)}\right),
$$

where $k(F)$ is the force-dependent off rate of the system, and $\dot{F}(F)$ is the force loading rate. In the simplest picture [12,13], the force-dependent off rate is given by [11]

$$
k(F)=k_{0} \exp \left(\frac{F \Delta x}{k_{\mathrm{B}} T}\right)
$$

where $k_{0}$ and $\Delta x$ are the zero-force off rate and distance to the free energy barrier, respectively. For a constant force loading rate $\dot{F}$ and an off rate from Eq. (2), the integral in Eq. (1) can be solved analytically [Fig. 1(a)]. Dudko 
(a)

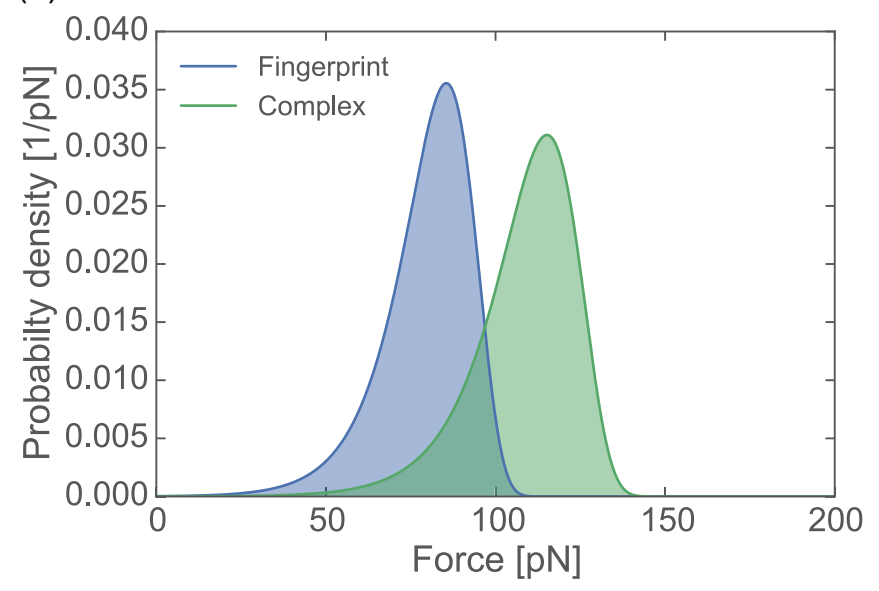

(b)

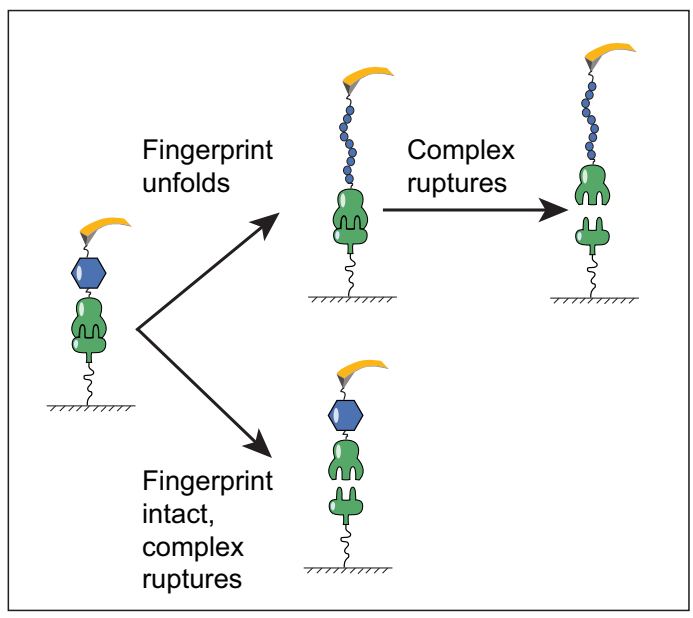

FIG. 1. (a) Overlapping distributions of fingerprint unfolding (blue region) and complex rupture (green region) for a constant loading rate $\dot{F}=200 \mathrm{pN} \mathrm{s}^{-1}$ with fingerprint $\Delta x=0.4 \mathrm{~nm}$, and $k_{0}=0.005 \mathrm{~s}^{-1}$ and complex parameters $\Delta x=0.35 \mathrm{~nm}$, and $k_{0}=$ $0.001 \mathrm{~s}^{-1}$ obtained by integrating Eq. (1) using Eq. (2). (b) Schematic of possible outcomes for the situation in (a). Analyzable data show fingerprint unfolding followed by complex rupture (upper path). Due to the overlapping distributions for unfolding and rupture, complex rupture with an intact fingerprint is also possible (lower path).

et al. [15] have used the Kramers theory [16] to obtain a more sophisticated expression for the force-dependent off rate, which accounts for the specific shape of the free energy landscape. This approach also provides an analytical solution to Eq. (1) for a constant loading rate and includes the height of the free energy barrier $\Delta G$ as an additional parameter. Over the years, more theoretical models describing the distributions for domain unfolding and receptor-ligand dissociation have been developed [17-22] that can be applied to experimentally measured force distributions.

Since a constant force loading rate is required to obtain an analytical solution for the distribution of forces in a pulling experiment, force ramp measurements [23,24], where the external force is ramped linearly, are an elegant technique to study protein unfolding and receptor-ligand dissociation. In an experiment where a receptor-ligand system is used to probe the unfolding behavior of a protein fingerprint domain of interest, care has to be taken when analyzing the resulting unfolding or rupture force distributions. If the probability distributions for fingerprint domain unfolding and complex rupture are disjunct, i.e., the complex ruptures at forces well above those required for fingerprint unfolding, the measured distributions are unbiased and can be readily analyzed using a desired form of Eq. (1). If those distributions have a substantial overlap [Fig. 1(a)], however, biasing effects have to be taken into account.

To pass the data analysis filter a given curve is required to exhibit both fingerprint unfolding and complex rupture [Fig. 1(b); upper path], i.e., the complex must not rupture prior to fingerprint unfolding [Fig. 1(b); lower path]. Consequently, the resulting distribution of fingerprint unfolding forces will be shifted downwards towards lower forces. By the same logic, experimentally observed distributions for receptor-ligand complex rupture forces will be truncated at the lower end and shifted upwards toward higher forces in a constant-loading-rate experiment. This biasing effect has been used qualitatively by Jobst et al. [8] to unambiguously identify a redundant dual binding mode in a receptor-ligand complex.

Here we treat this biasing effect quantitatively and calculate these effects independent of the model used in Eq. (1). For overlapping distributions of fingerprint, $p_{\mathrm{f}}(F)$, and receptorligand complex, $p_{\mathrm{c}}(F)$, the biased distribution of the fingerprint, $p_{\mathrm{f}}^{\star}(F)$, can be calculated by multiplying the original distribution by the cumulative probability for the bond to rupture at higher forces and renormalizing,

$$
p_{\mathrm{f}}^{\star}(F)=\frac{p_{\mathrm{f}}(F) \int_{F}^{\infty} \mathrm{d} F^{\prime} p_{\mathrm{c}}\left(F^{\prime}\right)}{\eta}
$$

where $\eta$ is a normalization constant. Since $p_{\mathrm{c}}(f)$ is normalized, Eq. (3) can be rewritten,

$$
\begin{aligned}
p_{\mathrm{f}}^{\star}(F) & =\frac{p_{\mathrm{f}}(F)\left(1-\int_{0}^{F} \mathrm{~d} F^{\prime} p_{\mathrm{c}}\left(F^{\prime}\right)\right)}{\eta} \\
& =\frac{p_{\mathrm{f}}(F)\left(1-P_{\mathrm{c}}(F)\right)}{\eta},
\end{aligned}
$$

where $P_{\mathrm{c}}(F)$ is the cumulative distribution function of the complex rupture forces. The normalization constant $\eta$ can be calculated by integrating over the numerator in Eq. (5):

$$
\eta=1-\int_{0}^{\infty} \mathrm{d} F p_{\mathrm{f}}(F) P_{\mathrm{c}}(F) .
$$

Intuitively, the biased fingerprint distribution is normalized by the ratio of curves that exhibit fingerprint unfolding vs all rupture events. The above calculations apply for both constantloading-rate and constant-speed measurements. By the same logic, the biased distribution of observed complex ruptures for a constant loading rate can be calculated as

$$
p_{\mathrm{c}}^{\star}(F)=\frac{p_{\mathrm{c}}(F) P_{\mathrm{f}}(F)}{\eta} .
$$

Both biased distributions for fingerprint unfolding, $p_{\mathrm{f}}^{\star}(F)$, and complex ruptures, $p_{\mathrm{c}}^{\star}(F)$, are normalized by the same yield parameter $\eta$ since both distributions are extracted from the same curves in a given data set. For a mathematical proof, see Appendix A. We note that the biasing effect on complex rupture in the constant-speed case is more difficult to quantify. 


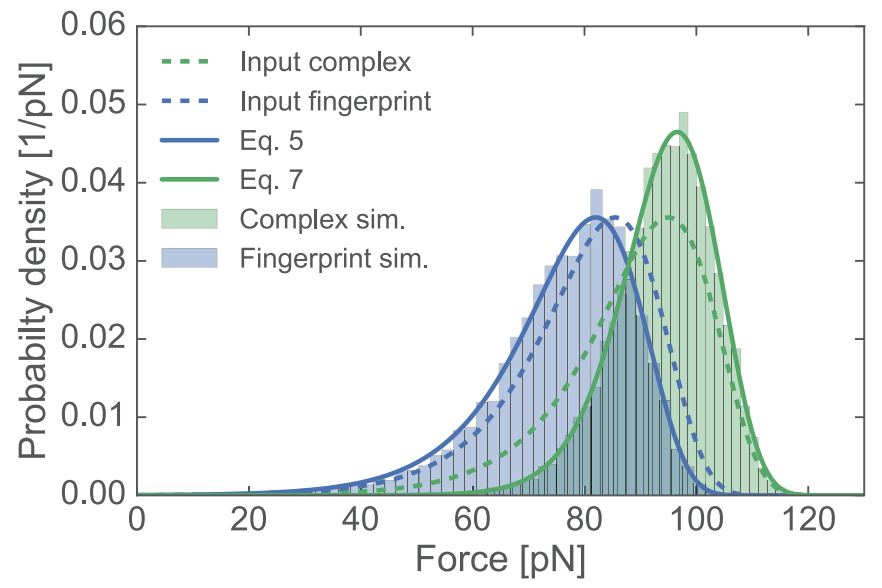

FIG. 2. Force ramp simulation with $\dot{F}=200 \mathrm{pN} \mathrm{s}^{-1}$, complex $\Delta x_{\mathrm{c}}=0.4 \mathrm{~nm}, k_{0, \mathrm{c}}=0.002 \mathrm{~s}^{-1}$, and fingerprint $\Delta x_{\mathrm{f}}=0.4 \mathrm{~nm}$, $k_{0, \mathrm{f}}=0.005 \mathrm{~s}^{-1}(\eta=0.71)$. Histograms of the simulated fingerprint unfolding forces and complex ruptures are shown in blue and green, respectively. Dashed blue and green lines represent the unbiased fingerprint unfolding and complex rupture force distributions, respectively. Biased unfolding and complex rupture force distributions for the fingerprint and complex calculated from Eqs. (5) and (7) are shown by solid blue and green lines, respectively.

Since the additional contour length released upon fingerprint unfolding is not immediately compensated for by a feedback on the force signal, there is a pronounced drop in force associated with fingerprint unfolding, giving rise to the characteristic sawtooth pattern in force extension traces. Ignoring the finite relaxation time of the harmonic pulling device, the force will drop from $F_{1}=F(x, L)$ to $F_{2}=F(x, L+\Delta L)$, where the former describes the (nonlinear) elastic behavior of the pulling device and potential linker molecules, and $\Delta L$ is the additional contour length released upon fingerprint unfolding. Whether or not the observed complex distribution is biased depends on the value of $p_{\mathrm{c}}\left(F_{2}\right)$. For $p_{\mathrm{c}}\left(F_{2}\right) \approx 0$, no biasing will occur, whereas $p_{\mathrm{c}}\left(F_{2}\right)>0$ will cause a biasing effect. In practice, $\Delta L$ is usually large enough to ensure that complex distributions are unbiased in constant-speed experiments, leaving a substantial bias only on the observed fingerprint distribution. A strategy to implement our correction procedure for the constant-speed protocol is proposed in Appendix B.

\section{MONTE CARLO SIMULATIONS}

To validate our results, we used a Monte Carlo approach to simulate fingerprint domain unfolding in combination with receptor-ligand dissociation in a constant-loading-rate protocol. Our simulation routine is similar to the approach described in Ref. [25] and uses the phenomenological model due to its analytical tractability. Briefly, we integrate Eq. (2) over a time step $\Delta t$, where $F=F(t)=\dot{F} t$, to obtain probabilities $p_{\mathrm{u}}$ and $p_{\mathrm{r}}$ for fingerprint unfolding and complex rupture, respectively. These probabilities are compared to independent random numbers $n_{\mathrm{u}}$ and $n_{\mathrm{r}}$ between 0 and unity. If $p_{\mathrm{u}}<n_{\mathrm{u}}$ and $p_{\mathrm{r}}<n_{\mathrm{r}}$, the fingerprint and complex remain intact and the next iteration is started. If $p_{\mathrm{u}}>n_{\mathrm{u}}$ and $p_{\mathrm{r}}<n_{\mathrm{r}}$, the corresponding force is noted as the fingerprint unfolding force and the next iteration is started with only the complex intact. The simulation then continues until $p_{\mathrm{r}}>n_{\mathrm{r}}$ and the corresponding force is noted as the complex rupture force. If $p_{\mathrm{u}}<n_{\mathrm{u}}$ and $p_{\mathrm{r}}>n_{\mathrm{r}}$ or $p_{\mathrm{u}}>n_{\mathrm{u}}$ and $p_{\mathrm{r}}>n_{\mathrm{r}}$, the complex ruptured prior to fingerprint unfolding or at the same time, and an experimental curve would be unanalyzable and filtered out during the data analysis procedure.

Results from a Monte Carlo simulation at constant loading rate $\dot{F}=200 \mathrm{pN} \mathrm{s}^{-1}$ with overlapping distributions for fingerprint unfolding and complex rupture are shown in Fig. 2. $N=10000$ traces were simulated and the observed fingerprint domain unfolding forces and receptor-ligand complex rupture forces were histogrammed. We only analyzed curves that showed both fingerprint unfolding and complex rupture to mimic experimental conditions. As expected, both fingerprint unfolding and complex rupture distributions (blue and green histograms in Fig. 2) are shifted from the unbiased input distributions (dashed blue and green lines in Fig. 2). The biased results are well described by our theoretical predictions, which are shown as solid blue and green lines in Fig. 2. To illustrate the potential errors that can occur from not accounting for the fingerprint biasing effect, we used the uncorrected distribution [Eq. (1)] to fit the biased fingerprint domain unfolding and complex rupture histograms and compared the resulting fit parameters $\Delta x_{\text {fit }}$ and $k_{0 \text {,fit }}$ with the unbiased input parameters (Table I). We found that for fingerprint domain unfolding, $\Delta x$ is hardly affected, while $k_{0}$ is overestimated by $30 \%$. For complex rupture $\Delta x$ is overestimated by $29 \%$ due to the smaller width of the biased distribution, while $k_{0}$ is underestimated by over an order of magnitude. If the unbiased parameters for the complex distribution are known from a control experiment, our predicted biased distributions can be used to fit the experimental data to obtain unbiased values for the fit parameters pertaining to the fingerprint, or vice versa. Alternatively, a global fitting procedure can be applied to both biased distributions for constant-loading-rate data to obtain unbiased fit parameters without prior knowledge of either distribution. Using this approach, we obtained global fit parameters that were within $6 \%$ of the input parameters (Table I).

TABLE I. Input vs fit parameters of the simulation shown in Fig. 2. For these parameters, the yield parameter equals $\eta=0.71$. Initially, the simulated distributions were fit with the uncorrected distributions ( $k_{0, \text { fit }}$ and $\left.\Delta x_{\text {fit }}\right)$. To correct for the biasing effects, both fingerprint and complex data were fit with their respective biased distributions [Eqs. (5) and (7)] in a global fitting procedure to obtain the corrected parameters $k_{0, \text { global }}$ and $\Delta x_{\text {global }}$.

\begin{tabular}{lcccrrr}
\hline \hline & $k_{0, \text { input }}\left(\mathrm{s}^{-1}\right)$ & $k_{0, \text { fit }}\left(\mathrm{s}^{-1}\right)$ & $k_{0, \text { global }}\left(\mathrm{s}^{-1}\right)$ & $\Delta x_{\text {input }}(\mathrm{nm})$ & $\Delta x_{\text {fit }}(\mathrm{nm})$ & $\Delta x_{\text {global }}(\mathrm{nm})$ \\
\hline Fingerprint & $5.0 \times 10^{-3}$ & $(6.6 \pm 0.2) \times 10^{-3}$ & $(4.7 \times 0.4) \times 10^{-3}$ & 0.400 & $0.402 \pm 0.007$ & $0.401 \pm 0.005$ \\
Complex & $2.0 \times 10^{-3}$ & $(0.10 \pm 0.02) \times 10^{-3}$ & $(1.9 \pm 0.02) \times 10^{-3}$ & 0.400 & $0.527 \pm 0.007$ & $0.402 \pm 0.005$ \\
\hline \hline
\end{tabular}




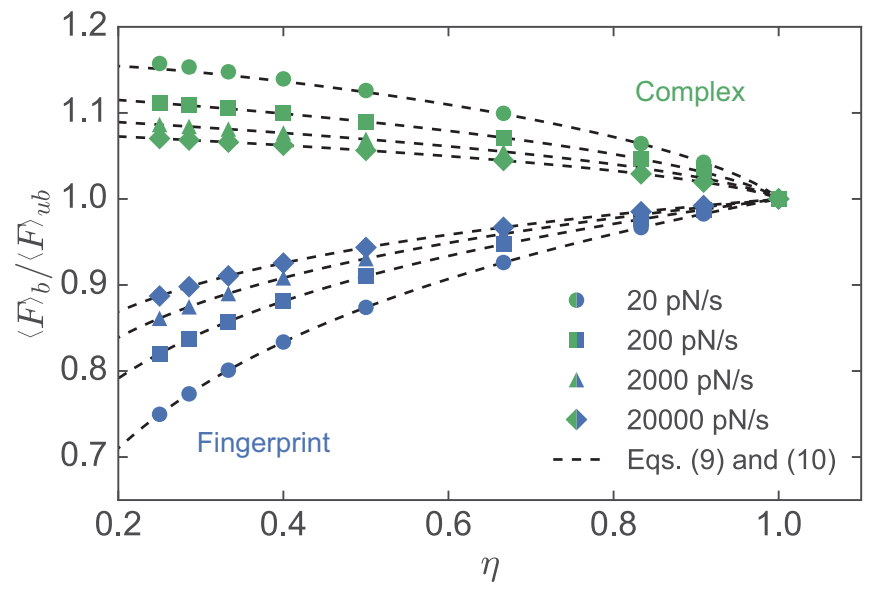

FIG. 3. Biasing of fingerprint unfolding and complex rupture forces as a function of the theoretical yield of curves exhibiting fingerprint unfolding $\eta$. Data points show the relative mean biased unfolding force $\langle F\rangle_{\mathrm{b}} /\langle F\rangle_{\mathrm{ub}}$; fingerprint and complex data are shown in blue and green, respectively. For fingerprint data, parameters were held constant at $\Delta x_{\mathrm{f}}=0.4 \mathrm{~nm}$ and $k_{0, \mathrm{f}}=0.005 \mathrm{~s}^{-1}$, while the complex distribution was shifted by maintaining $\Delta x_{\mathrm{c}}=0.4 \mathrm{~nm}$ and varying the off rate. For complex data, $\Delta x_{\mathrm{c}}=0.4 \mathrm{~nm}$ and $k_{0, \mathrm{c}}=0.005 \mathrm{~s}^{-1}$ were held constant and the fingerprint distribution was shifted by maintaining $\Delta x_{\mathrm{f}}=0.4 \mathrm{~nm}$ and varying the off rate. Dashed lines represent predictions based on Eq. (9) and (10).

\section{SPECIAL CASE: EQUAL POTENTIAL WIDTHS}

In Fig. 3 we quantify the magnitude of the biasing effect by numerically calculating the relative mean biased unfolding force of the fingerprint (blue symbols) and complex (green symbols) $\langle F\rangle_{\mathrm{b}} /\langle F\rangle_{\mathrm{ub}}=\int \mathrm{d} F F p_{\mathrm{f} / \mathrm{c}}^{*}(F) / \int \mathrm{d} F F p_{\mathrm{f} / \mathrm{c}}(F)$ as a function of the theoretical ratio of curves exhibiting fingerprint unfolding prior to receptor-ligand complex rupture, $\eta$ [Eq. (6)]. For analytical tractability we chose the special case $\Delta x_{\mathrm{c}}=\Delta x_{\mathrm{f}}$. With this simplification we find for the fingerprint

$$
\langle F\rangle_{\mathrm{b}}=\frac{k_{\mathrm{B}} T}{\Delta x_{\mathrm{f}}} e^{\frac{k_{\mathrm{B}} T k_{0, \mathrm{f}}}{\Delta x_{\mathrm{f}} \eta}} E_{1}\left(\frac{k_{\mathrm{B}} T k_{0, \mathrm{f}}}{\Delta x_{\mathrm{f}} r \eta}\right),
$$

where $E_{1}(x)$ is the exponential integral. Using $e^{x} E_{1}(x) \cong$ $\ln \left(1+\frac{e^{-\gamma}}{x}\right)$ we can produce an analytical approximation for the relative mean biased unfolding force for the aforementioned special case,

$$
\frac{\langle F\rangle_{\mathrm{b}}}{\langle F\rangle_{\mathrm{ub}}} \cong \frac{\ln \left(1+\frac{r \Delta x_{\mathrm{f}}}{k_{0, \mathrm{f}} k_{\mathrm{B}} T} e^{-\gamma} \eta\right)}{\ln \left(1+\frac{r \Delta x_{\mathrm{f}}}{k_{0, \mathrm{f}} k_{\mathrm{B}} T} e^{-\gamma}\right)},
$$

where $\gamma=0.577 \ldots$ is the Euler-Mascheroni constant. The analogous result for the complex reads

$$
\frac{\langle F\rangle_{\mathrm{b}}}{\langle F\rangle_{\mathrm{ub}}} \cong \frac{1}{\eta}-\frac{\ln \left(1+\frac{r \Delta x_{\mathrm{c}}}{k_{0, \mathrm{c}} k_{\mathrm{B}} T} e^{-\gamma}(1-\eta)\right)}{\ln \left(1+\frac{r \Delta x_{\mathrm{c}}}{k_{0, \mathrm{c}} k_{\mathrm{B}} T} e^{-\gamma}\right)} \frac{1-\eta}{\eta} .
$$

For this special case Eq. (6) can be evaluated analytically, yielding $\eta=\left(1+\frac{k_{0, \mathrm{c}}}{k_{0, \mathrm{f}}}\right)^{-1}$. Equations (9) and (10) (dashed lines in Fig. 3) agree very well with our numerical results over the loading-rate regime covered. Figure 3 clearly shows that the biasing effect is more pronounced for low loading rates, consistent with our theoretical predictions based on
Eqs. (9) and (10). In cases where data cannot be corrected for a potential biasing effect, e.g., due to low experimental yields, the magnitude of the biasing effect can be minimized by increasing the loading rate in a pulling experiment.

\section{CONCLUSION}

Our calculations provide a framework for analyzing singlemolecule force spectroscopy data where receptor-ligand systems are used as specific handles to study a fingerprint domain of interest, or vice versa. In such experiments, it is many times the case that the distributions of fingerprint domain unfolding and complex rupture have a significant overlap (a few exemplary cases can be found in Refs. [3,9,10,26]). In this case biasing effects will occur and should be considered in the analysis procedure. Our findings can be applied to both constant-speed and force ramp (constant-loading-rate) experimental protocols, however, it should be noted that the biasing effect on complex unbinding is more complicated in the constant-speed protocol, due to the drop in force upon fingerprint unfolding dependent on the length of the unfolded domain. Since the biasing effects are solely due to the statistical nature of domain unfolding or complex unbinding, our results, specifically Eqs. (5) and (7), are valid regardless of the specific model used in Eq. (1).

\section{ACKNOWLEDGMENTS}

The authors would like to thank Ellis Durner, Markus A. Jobst, Magnus S. Bauer, Fabian Baumann, and Steffen Sedlak for valuable discussions. This work was supported by an advanced grant to H.E.G. from the European Research Council (Cellufuel Grant No. 294438). M.A.N. acknowledges funding from Society in Science The Branco Weiss Fellowship program administered by ETH Zürich, Switzerland.

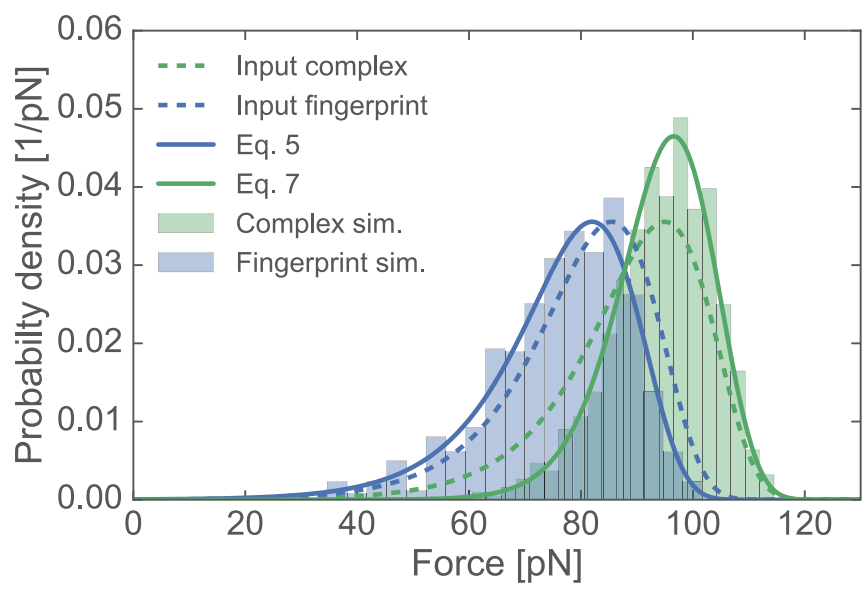

FIG. 4. Force ramp simulation with $\dot{F}=200 \mathrm{pN} \mathrm{s}^{-1}$, complex $\Delta x_{\mathrm{c}}=0.4 \mathrm{~nm}, k_{0, \mathrm{c}}=0.002 \mathrm{~s}^{-1}$, fingerprint $\Delta x_{\mathrm{f}}=0.4 \mathrm{~nm}, k_{0, \mathrm{f}}=$ $0.005 \mathrm{~s}^{-1}(\eta=0.71)$, and simulated traces $N=1000$. Histograms of the simulated fingerprint unfolding forces and complex ruptures are shown in blue and green, respectively. Dashed blue and green lines represent the unbiased fingerprint unfolding and complex rupture force distributions, respectively. Biased unfolding and complex rupture force distributions for the fingerprint and complex calculated from Eqs. (5) and (7) are shown by solid blue and green lines, respectively. 
TABLE II. Input vs fit parameters of the simulation shown in Fig. 4. For these parameters, the yield parameter equals $\eta=0.71$. Initially, the simulated distributions were fit with the uncorrected distributions ( $k_{0, \text { fit }}$ and $\left.\Delta x_{\mathrm{fit}}\right)$. To correct for the biasing effects, both fingerprint and complex data were fit with their respective biased distributions [Eqs. (5) and (7)] in a global fitting procedure to obtain the corrected parameters $k_{0, \text { global }}$ and $\Delta x_{\text {global }}$.

\begin{tabular}{|c|c|c|c|c|c|c|}
\hline & $k_{0, \text { input }}\left(\mathrm{s}^{-1}\right)$ & $k_{0, \text { fit }}\left(\mathrm{s}^{-1}\right)$ & $k_{0, \text { global }}\left(\mathrm{s}^{-1}\right)$ & $\Delta x_{\text {input }}(\mathrm{nm})$ & $\Delta x_{\text {fit }}(\mathrm{nm})$ & $\Delta x_{\text {global }}(\mathrm{nm})$ \\
\hline Fingerprint & $5.0 \times 10^{-3}$ & $(5.9 \pm 1.2) \times 10^{-3}$ & $(4.3 \pm 1.5) \times 10^{-3}$ & 0.400 & $0.409 \pm 0.011$ & $0.410 \pm 0.020$ \\
\hline Complex & $2.0 \times 10^{-3}$ & $(0.2 \pm 0.1) \times 10^{-3}$ & $(2.7 \pm 1.3) \times 10^{-3}$ & 0.400 & $0.504 \pm 0.020$ & $0.384 \pm 0.021$ \\
\hline
\end{tabular}

\section{APPENDIX A: IDENTITY OF YIELD PARAMETER $\eta$ IN EQS. (5) AND (7)}

As we state in the text, the normalization parameter $\eta$ is equal to the ratio of curves that exhibit fingerprint unfolding vs all rupture events. In other words a fraction $1-\eta$ of all curves will be "missed" in an actual experiment, since they do not exhibit fingerprint unfolding and would hence be discarded during data analysis. Consequently, both fingerprint and complex distributions (which are obtained from the same curves) need to be normalized by the same $\eta$ to obtain probability distributions normalized to unity. Mathematically, one needs to show that

$$
1-\int_{0}^{\infty} \mathrm{d} F p_{\mathrm{f}}(F) P_{\mathrm{c}}(F)=\eta=\int_{0}^{\infty} \mathrm{d} F p_{\mathrm{c}}(F) P_{\mathrm{f}}(F) .
$$

We use integration by parts and $\int_{0}^{F} \mathrm{~d} F^{\prime} p\left(F^{\prime}\right)=P(F)$ to evaluate the right-hand side (rhs) of Eq. (A1):

$$
\text { rhs }=\left.P_{\mathrm{c}}(F) P_{\mathrm{f}}(F)\right|_{0} ^{\infty}-\int_{0}^{\infty} \mathrm{d} F P_{\mathrm{c}}(F) p_{\mathrm{f}}(F) .
$$

Since $P(0)=0$ and $P(\infty)=1$ this is equal to the left-hand side of Eq. (A1). (a)

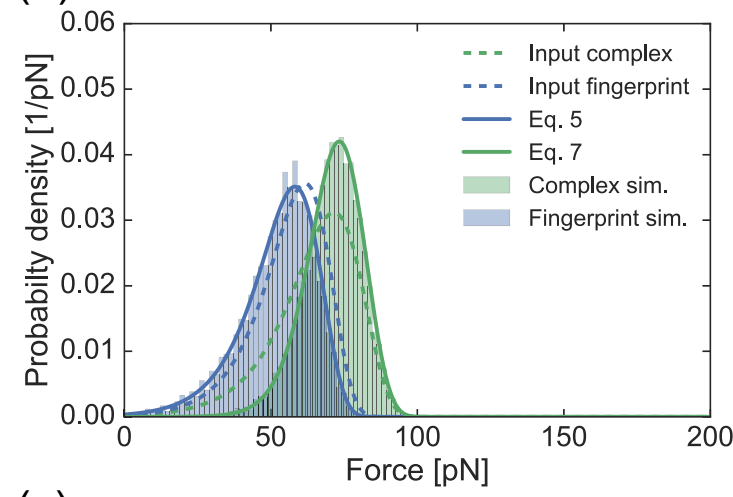

(c)

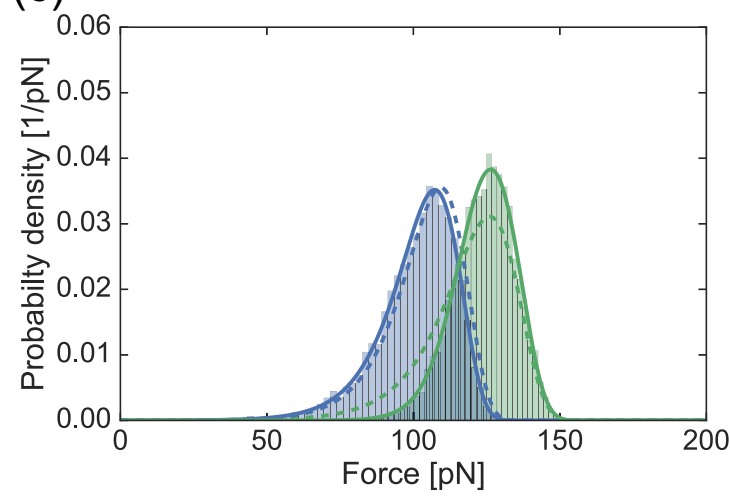

(b)

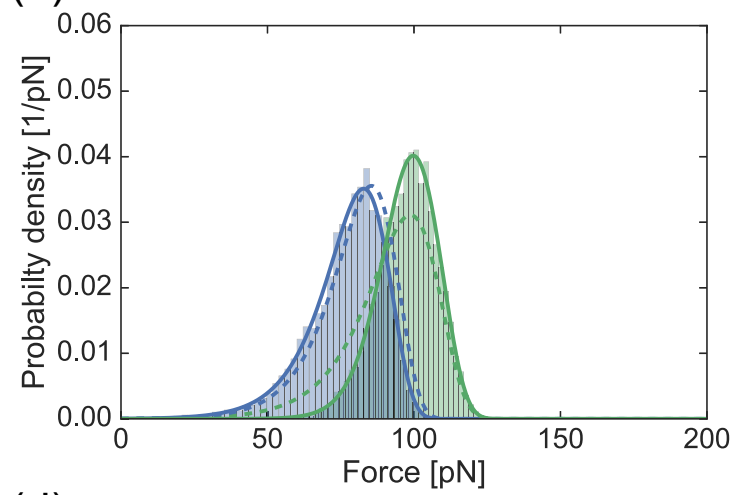

(d)

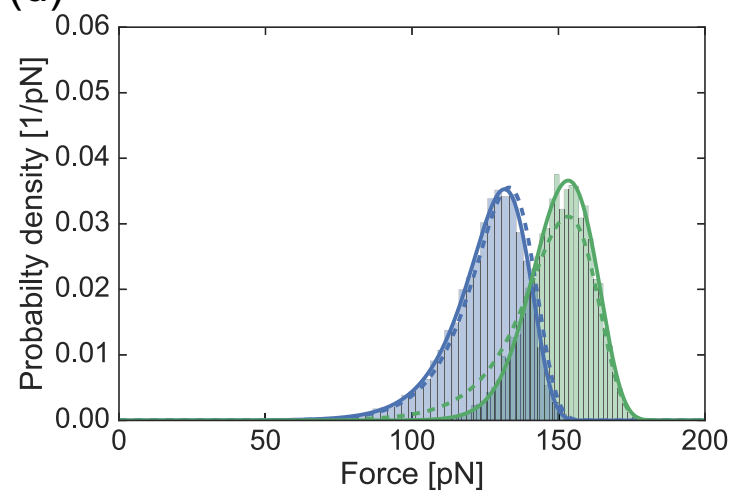

FIG. 5. Force ramp simulation with complex $\Delta x_{\mathrm{c}}=0.35 \mathrm{~nm}, k_{0, \mathrm{c}}=0.004 \mathrm{~s}^{-1}$, fingerprint $\Delta x_{\mathrm{f}}=0.4 \mathrm{~nm}, k_{0, \mathrm{f}}=0.005 \mathrm{~s}^{-1}$, and varying $\dot{F}$. Due to the different potential widths $\Delta x$, the yield parameter $\eta$ changes with the loading rate. Values of the loading rate and resulting yield parameter are (a) $\dot{F}=20 \mathrm{pN} \mathrm{s}^{-1}$ and $\eta=0.70$, (b) $\dot{F}=200 \mathrm{pN} \mathrm{s}^{-1}$ and $\eta=0.75$, (c) $\dot{F}=2000 \mathrm{pN} \mathrm{s}^{-1}$ and $\eta=0.80$, and (d) $\dot{F}=$ $20000 \mathrm{pN} \mathrm{s}^{-1}$ and $\eta=0.85$. Histograms of the simulated fingerprint unfolding forces and complex ruptures are shown in blue and green, respectively. Dashed blue and green lines represent the unbiased fingerprint unfolding and complex rupture force distributions, respectively. Biased unfolding and complex rupture force distributions for the fingerprint and complex calculated from Eqs. (5) and (7) are shown by solid blue and green lines, respectively. 


\section{APPENDIX B: CONSTANT SPEED}

As pointed out in the text, the correction procedure for distributions obtained from constant-speed measurements is more involved. In this Appendix we discuss a strategy for extracting unbiased parameters from fingerprint distributions for this pulling protocol. Due to the nonlinear elasticity of linker molecules (e.g., polyethylene glycol, spacers, or unfolded protein backbone), the force loading rate $\dot{F}=\dot{F}(F)$ becomes a function of the force and the integral in Eq. (1) can no longer be evaluated analytically. In a standard pulling experiment, a harmonic pulling device (e.g., atomic force microscopy cantilever or optically trapped bead) is connected to the aforementioned linker molecules. By applying a force balance it can be shown that the force loading rate is given by [14]

$$
\frac{v}{\dot{F}(F)}=\frac{1}{k_{\mathrm{h}}}+\frac{\partial x(F)}{\partial F},
$$

where $v$ is the pulling speed, $k_{\mathrm{h}}$ is the spring constant of the harmonic pulling device, and $x(F)$ is the force-dependent extension of the linker. The biased distribution for fingerprint unfolding $p_{\mathrm{f}}^{\star}(F)$ can be computed by numerically solving the integrals occurring in Eqs. (5) and (6), using a model for the elastic response of the linker molecules such as the worm-like-chain model, freely rotating model, or composite model proposed by Livadaru et al. [27] in Eq. (B1). The choice of model should be made based on the force regime in which fingerprint unfolding and complex rupture are expected and the molecular linkers present in an experimental setup need to be accounted for in these models via their contour length, $L$, and elasticity, e.g., persistence length $p$. The force-dependent loading rate for the worm-like-chain model has been derived as Eq. (4) in Ref. [14].

\section{APPENDIX C: SUPPLEMENTAL FIGURES AND TABLES}

This Appendix contains contains two figures and one table that support the results in the text. Figure 4 shows the results of a simulation with parameters identical to those in Fig. 2, except only $N=1000$ traces were simulated, to mimic a total number of curves more similar to average experimental yields. The extracted fit parameters for this simulation are listed in Table II. We note that despite the increase in uncertainties, the extracted parameters from our global fitting procedure still reproduce the input parameters much better than those obtained from a fit to the uncorrected distributions. Figure 5 shows the results of Monte Carlo simulations at different loading rates ranging from $\dot{F}=20 \mathrm{pN} \mathrm{s}^{-1}$ to $\dot{F}=20000 \mathrm{pN} \mathrm{s}^{-1}$. Due to the different potential widths $\Delta x_{\mathrm{c}}=0.35 \mathrm{~nm}$ and $\Delta x_{\mathrm{f}}=0.4 \mathrm{~nm}$, the yield parameter $\eta$ varies for the different simulations.
[1] J. A. Rivas-Pardo, E. C. Eckels, I. Popa, P. Kosuri, W. A. Linke, and J. M. Fernández, Cell Reports 14, 1339 (2016).

[2] A. del Rio, R. Perez-Jimenez, R. Liu, P. Roca-Cusachs, J. M. Fernandez, and M. P. Sheetz, Science 323, 638 (2009).

[3] S. W. Stahl, M. A. Nash, D. B. Fried, M. Slutzki, Y. Barak, E. A. Bayer, and H. E. Gaub, Proc. Natl. Acad. Sci. USA 109, 20431 (2012).

[4] C. Schoeler, K. H. Malinowska, R. C. Bernardi, L. F. Milles, M. A. Jobst, E. Durner, W. Ott, D. B. Fried, E. A. Bayer, K. Schulten, H. E. Gaub, and M. A. Nash, Nature Commun. 5, 5635 (2014).

[5] W. Ott, M. A. Jobst, C. Schoeler, H. E. Gaub, and M. A. Nash, J. Struct. Biol. (2016), doi: 10.1016/j.jsb.2016.02.011.

[6] K. C. Neuman and A. Nagy, Nature Methods 5, 491 (2008).

[7] S. B. Smith, L. Finzi, and C. Bustamante, Science 258, 1122 (1992).

[8] M. A. Jobst, L. F. Milles, C. Schoeler, W. Ott, D. B. Fried, E. A. Bayer, H. E. Gaub, and M. A. Nash, eLife 4, e10319 (2015).

[9] F. Baumann, M. S. Bauer, L. F. Milles, A. Alexandrovich, H. E. Gaub, and D. A. Pippig, Nature Nanotechnol. 11, 89 (2016).

[10] M. Otten, W. Ott, M. A. Jobst, L. F. Milles, T. Verdorfer, D. A. Pippig, M. A. Nash, and H. E. Gaub, Nature Methods 11, 1127 (2014).

[11] G. Bell, Science 200, 618 (1978).

[12] S. Izrailev, S. Stepaniants, M. Balsera, Y. Oono, and K. Schulten, Biophys. J. 72, 1568 (1997).

[13] E. Evans and K. Ritchie, Biophys. J. 72, 1541 (1997).
[14] O. K. Dudko, G. Hummer, and A. Szabo, Proc. Natl. Acad. Sci. USA 105, 15755 (2008).

[15] O. K. Dudko, G. Hummer, and A. Szabo, Phys. Rev. Lett. 96, 108101 (2006).

[16] H. A. Kramers, Physica 7, 284 (1940).

[17] O. K. Dudko, Q. Rev. Biophys. 49, e3 (2015).

[18] B. Heymann and H. Grubmüller, Phys. Rev. Lett. 84, 6126 (2000).

[19] J. T. Bullerjahn, S. Sturm, and K. Kroy, Nature Commun. 5, 4463 (2014).

[20] R. W. Friddle, Phys. Rev. Lett. 100, 138302 (2008).

[21] R. W. Friddle, A. Noy, and J. J. De Yoreo, Proc. Natl. Acad. Sci. USA 109, 13573 (2012).

[22] T. Hugel, M. Rief, M. Seitz, H. E. Gaub, and R. R. Netz, Phys. Rev. Lett. 94, 048301 (2005).

[23] A. F. Oberhauser, P. K. Hansma, M. Carrión-Vázquez, and J. M. Fernandez, Proc. Natl. Acad. Sci. USA 98, 468 (2001).

[24] E. Evans, A. Leung, V. Heinrich, and C. Zhu, Proc. Natl. Acad. Sci. USA 101, 11281 (2004).

[25] C. Friedsam, A. K. Wehle, F. Kühner, and H. E. Gaub, J. Phys.: Condens. Matter 15, S1709 (2003).

[26] J. P. Mueller, S. Mielke, A. Loef, T. Obser, C. Beer, L. K. Bruetzel, D. A. Pippig, W. Vanderlinden, J. Lipfert, R. Schneppenheim, and M. Benoit, Proc. Natl. Acad. Sci. USA 113, 1208 (2016).

[27] L. Livadaru, R. R. Netz, and H. J. Kreuzer, Macromolecules 36, 3732 (2003). 